\title{
EL SISTEMA MONETARIO INTERNACIONAL Y SUS PERSPECTIVAS DE FUTURO
}

\author{
FÉLIX VARELA PARACHE \\ Universidad de Alcalá de Henares \\ MONTSERRAT HINAREJOS ROJO \\ Universidad de Alcalá de Henares
}

\begin{abstract}
RESUMEN
Este artículo parte de analizar la evolución del sistema monetario internacional desde la introducción de los tipos de cambio flotantes en 1973 hasta la actualidad y la controversia a que ha dado lugar el comportamiento del régimen de flexibilidad cambiaria, así como la conveniencia o no de establecer un sistema de tipos de cambio más estable. Después de hacer referencia a algunas de las propuestas que se han formulado para dotar de más estabilidad al sistema, se analiza la influencia de la mayor movilidad de los capitales y las enseñanzas que en este sentido puede ofrecer la reciente crisis del Sistema Monetario Europeo.
\end{abstract}

\section{ABSTRACT}

This article analyses the evolution of the international monetary system from the introduction of floating exchange rates in 1973 to the present. It also dwells on the controversy over the results of a flexible exchange rate system versus a fixed exchange regime. Later it studies the proposals put forward for the stability of the system, the influence of capital movements and the recent events of the crisis of the European Monetary System.

\section{INTRODUCCIÓN}

Hay una corriente de pensamiento que ve la evolución del sistema monetario internacional como la sucesión de una serie de períodos en que predomina la existencia de reglas - tipos de cambio fijos- y otros en que se da preferencia a la dis- 
crecionalidad, adoptándose un sistema de tipos de cambio más o menos libre. En este esquema, el sistema monetario actual representaría el extremo de la máxima discrecionalidad, ya que prácticamente no impone obligación alguna a los países. Algunos autores consideran que se trata más bien de un «no-sistema», ya que deja a la libre opción de los países tanto el sistema de fijación de los tipos de cambio, como la combinación de medidas de ajuste y financiación que aquéllos pueden aplicar para hacer frente a los problemas de balanza de pagos.

Las fuertes oscilaciones en los tipos de cambio durante las últimas décadas y el desencanto sobre su eficacia como mecanismo de ajuste, han llevado a muchos economistas a desear un sistema monetario más estable y reglamentado. Surgieron así diversas propuestas encaminadas a este propósito. La experiencia favorable del Sistema Monetario Europeo en los años ochenta y el inicio de los noventa, pareció dar nuevo impulso a esta corriente de pensamiento. Sin embargo, la crisis monetaria europea de 1992-93 ha puesto de relieve la dificultad de comprometerse en mecanismos de estabilización de los tipos de cambio, con plena libertad de capitales, a menos que los países estén dispuestos a renunciar a la independencia de su política monetaria.

La libertad de los movimientos de capital no es patrimonio exclusivo de los países europeos que han conformado el Mercado Único. Por el contrario, dicha liberalización ha tenido un carácter global y se ha extendido no sólo entre los países industriales, sino también a un grupo cualitativamente importante de países en desarrollo. La liberalización de los flujos financieros y la rápida expansión de los movimientos privados de fondos, constituyen características importantes de la evolución reciente y plantean una nueva situación para el equilibrio de los mercados de cambios y para el objetivo de conseguir una mayor estabilidad de los tipos de cambio.

Este tema constituirá el principal centro de atención del presente trabajo. Para ello, en el apartado 2 se realiza una breve panorámica sobre el establecimiento del sistema de Bretton Woods, sus características y su funcionamiento hasta su ruptura. En el apartado 3 se analizará la evolución del sistema monetario internacional que lleva a la flotación y se señalarán algunas de las razones por las que ésta no ha respondido a las expectativas favorables que muchos economistas habían depositado en ella. En el apartado 4 se resumirán las propuestas más importantes que se han realizado para dotar al sistema de mayor estabilidad. En el apartado 5 nos referiremos a la modificación fundamental que la liberalización de los movimientos de capital y la globalización de los mercados financieros introduce en el funcionamiento del sistema. Para ello resulta de gran interés la experiencia derivada de la crisis monetaria europea. En el último apartado se establecerán las conclusiones que se derivan del análisis anterior. 


\section{EL SISTEMA DE BRETTON WOODS}

El sistema monetario internacional nacido de la Conferencia de Bretton Woods de 1944 fue la respuesta de la comunidad internacional a los problemas monetarios y financieros que se vivieron durante la década de los treinta, consecuencias de las medidas tomadas individualmente por los países en un intento de salvaguardar sus economías internas ante los efectos de la Gran Depresión.

Los rasgos característicos del panorama monetario de la década de los treinta serían los que el nuevo orden económico de la posguerra trataría de evitar a toda costa: incertidumbre introducida por los tipos de cambio fluctuantes tras el abandono del Patrón Oro y utilización de los tipos de cambio para finalidades comerciales de «empobrecimiento al vecino». Otras actuaciones relevantes en el período fueron las restricciones sobre la compra y venta de monedas extranjeras y el bilateralismo en los pagos internacionales instrumentado a través de acuerdos de clearing bilateral, forma de pago que si bien salva los obstáculos impuestos por los controles de cambio también ejerce graves efectos restrictivos sobre el comercio internacional.

Es evidente que estas soluciones nacionalistas e individuales adoptadas por los países durante la década de los treinta tuvieron una serie de consecuencias económicas negativas para la economía internacional. Al final del conflicto bélico era necesario fomentar un entorno económico internacional armonioso, en el que la cooperación entre los países evitara la adopción de políticas perniciosas para el crecimiento y desarrollo económico del conjunto de las naciones. Para ello habría de establecerse un nuevo sistema monetario internacional que tendría muy en cuenta la historia económica de la década anterior al comienzo de la segunda guerra mundial. Este nuevo sistema fue forjado gracias a las ideas sobre el orden económico de la posguerra que plasmaron en sendos planes un británico y un americano, John Maynard Keynes y Harry Dexter White, respectivamente, a principios de los años cuarenta. El resultado de lo negociado fue presentado en la Conferencia Monetaria y Financiera de las Naciones Unidas celebrada en Bretton Woods en julio de 1944, dando lugar al establecimiento del Fondo Monetario Internacional y del Banco Internacional de Reconstrucción y Desarrollo, hoy en día, Banco Mundial. Estos organismos conocidos como «las instituciones de Bretton Woods» formaron la estructura básica del sistema económico y financiero internacional de la posguerra.

El Fondo Monetario Internacional se constituyó como el pilar central del nuevo sistema monetario internacional, cuyos fines claramente definidos en el artículo primero de su convenio constitutivo diseñaban un auténtico código de 
conducta para el mantenimiento de la estabilidad cambiaria y el desarrollo del comercio internacional sin restricciones a los pagos. Surgía así el llamado sistema de paridades o sistema de Bretton Woods, basado en la existencia de tipos de cambio fijos pero ajustables, combinando de esta manera la estabilidad de los tipos de cambio en el corto plazo con la posibilidad de modificarlos ocasionalmente en el largo plazo si la situación económica del país así lo exigía.

El sistema se basaba en la fijación por cada país de una paridad, puramente nominal, de su moneda en términos de oro o en relación con el dólar de Estados Unidos del peso y valor vigentes en primero de julio de 1944. Los países tendrían la obligación de mantener sus tipos de cambio en una banda de fluctuación del $\pm 1 \%$ en torno a la paridad señalada. Sin embargo, esta obligación no era absoluta, pudiéndose modificar la paridad en caso de que el país incurriese en un «desequilibrio fundamental» y justificase el porcentaje de la devaluación que pretendía llevar a cabo. Si no se daban estas circunstancias, el mecanismo de ajuste para conseguir el equilibrio de la balanza de pagos operaría sobre las variables interiores, apoyado en los mecanismos de financiación creados por el FMI para tales casos, que consistían en un reforzamiento de las reservas de divisas del país a través de préstamos del Fondo.

La regulación de los tipos de cambio no fue el único objetivo del sistema creado en Bretton Woods, sino que sus propósitos se extendían a la eliminación de toda clase de discriminaciones monetarias y restricciones a los pagos internacionales motivados por operaciones corrientes. El artículo VIII del convenio constitutivo del FMI imponía la obligación de convertibilidad para las transacciones por cuenta corriente, admitiendo en su artículo XIV la posibilidad de establecer dicha convertibilidad después de un período transitorio de adaptación, al cual se acogieron la mayoría de los países. Habría que esperar hasta 1958 para que la prohibición de aplicar restricciones a los pagos corrientes se cumpliera de hecho en la mayor parte de los países industriales. La liberalización de las transacciones corrientes irán acompañadas en los años sesenta por una progresiva liberalización de capitales, que nunca fue exigida por el FMI y que pondría de manifiesto la debilidad del sistema de tipos de cambio fijos pero ajustables de Bretton Woods.

El funcionamiento del sistema en la práctica introdujo claras modificaciones respecto a lo acordado en Bretton Woods. Pronto se decantaría hacia un sistema dólar donde la política monetaria de los Estados Unidos condicionaría la del resto de los países y, por otra parte, el mecanismo de ajuste previsto a través de la modificación del tipo de cambio operaría sólo muy excepcionalmente en los paí- 
ses industriales a partir de 1950. El ajuste de los tipos de cambio fue conscientemente retrasado por las autoridades que trataron de evitar así los riesgos políticos asociados a la devaluación, tales como la pérdida de prestigio para el gobierno que la adoptaba y la reducida eficacia de la medida si era seguida por otros países, así como los riesgos de que se produjeran movimientos especulativos de capital tras la modificación de la paridad en situaciones futuras. De tal manera que la paulatina liberalización de los movimientos de capitales a lo largo de los años sesenta obligó a los países a renunciar a la devaluación como instrumento monetario puesto que su utilización debilitaba la capacidad del país para luchar contra la especulación. La pérdida del carácter ajustable del sistema se unía a su conversión, de hecho, en un sistema centrado en el dólar desde 1950, moneda en la que se mantenían las reservas que los países consiguieron acumular. El crecimiento de las reservas internacionales se hizo especialmente necesario a raíz de la expansión del comercio internacional durante la década de los cincuenta, siendo los Estados Unidos los únicos proveedores de liquidez del sistema, puesto que sólo el dólar tenía la capacidad de crecimiento necesaria para la expansión de las reservas internacionales.

La expansión de la liquidez a través de la creación de activos en dólares se realizó gracias al déficit de la balanza de pagos americana que no implicó ningún peligro para el sistema mientras el resto de los países iban acumulando las reservas en dólares que consideraban necesarias. Pero, en la segunda mitad de los años sesenta, cuando los pasivos en dólares de los Estados Unidos frente a entidades oficiales crecieron de tal manera que superaron ampliamente a las tenencias de oro y el déficit de la balanza de pagos americana se agudizó, fue cuando el resto de países dudó sobre la conveniencia de seguir acumulando dólares y procedieron a la conversión de esta moneda en oro. Dichos ataques especulativos provocaron presiones de los Estados Unidos sobre otros países para evitar la convertibilidad oro del dólar a partir de 1968. Con ello, los Estados Unidos conseguían evitar la única obligación de disciplina externa que mantenía con el sistema de Bretton Woods. En adelante, todo dependería de las exigencias internas de la política monetaria de los americanos que pronto empezaría a divergir con los intereses internacionales, al llevar a cabo a principios de los años setenta una política monetaria claramente inflacionista. El resultado final de la conjunción de los problemas que presentó el sistema de Bretton Woods en su funcionamiento fue el colapso del sistema entre 1971 y 1973, cuando los Estados Unidos abandonan definitivamente la convertibilidad oro del dólar, produciéndose la flotación de los tipos de cambio de las monedas, sistema que perdura hasta nuestros días. 


\section{EL SISTEMA DE FLOTACIÓN: ANTECEDENTES, FUNCIONAMIENTO Y DESILUSIONES}

\subsection{El sistema de Bretton Woods y su ruptura ${ }^{1}$}

El sistema de Bretton Woods concebido como un sistema de tipos de cambio fijos con carácter ajustable parecía conjuntar lo mejor del Patrón Oro, en cuanto a estabilidad, con las ventajas de los tipos de cambio flotantes, en cuanto a flexibilidad al permitir el ajuste de la paridad, no se reveló tan eficaz como se había previsto. Ya en su época, en los años sesenta, se señalaron dos asimetrias que provocaban una desigual distribución del peso del ajuste: entre países deficitarios y países con superávit, imponiendo el peso en los primeros; y entre país con moneda de reserva (Estados Unidos), resto de los países, que parecía eximir a aquél de las obligaciones de ajuste. También se observó en la época, que los ajustes tendieron generalmente a retrasarse, exigiendo después mayores y más drásticas medidas para conseguir el equilibrio. Pero quizá el principal defecto del mecanismo de ajuste diseñado en Bretton Woods fue no contar con un factor que sus redactores apenas tuvieron en cuenta: los movimientos de capital.

En el artículo VIII del Convenio Constitutivo del Fondo Monetario Internacional (FMI), se estableció el principio de la convertibilidad de las transacciones corrientes, pero no de capitales. Ésta parecía difícil de lograr en la perspectiva de aquellos años; además, tampoco era clara su deseabilidad después de la experiencia negativa de los años treinta. Pero la creciente liberalización de los pagos internacionales que se produjo con la declaración de convertibilidad externa de las monedas de los principales países, llevó, a partir de 1958, a extender tal liberalización a operaciones de capital. Aunque desigual e incompleta, tal liberalización fue suficiente para ejercer un efecto sobre las políticas de ajuste: la modificación de paridad pareció una medida especialmente indeseable, ya que quitaba credibilidad a la actuación del Gobierno y facilitaba los ataques especulativos futuros. No es extraño que los estudios realizados muestren que los principales países realizaron pocos ajustes de paridades y muy separados en el tiempo, frente a los numerosos y frecuentes de los países en desarrollo. Son precisamente los países que liberalizaron más sus flujos financieros, los que tuvieron que renunciar en mayor medida al tipo de cambio como instrumento de ajuste. Estos países sólo realizaron modificaciones de paridad en situaciones

' Se ha utilizado como base del epígrafe Varela (1994a), donde puede encontrarse un tratamiento más amplio del tema. 
muy excepcionales, $y$, muchas veces, cuando el grave desequilibrio causado por el Gobierno precedente, permitía atribuirle la culpabilidad última de la medida adoptada.

La limitada pero creciente liberalización de capitales hizo posible una serie de crisis monetarias que afectaron a diversas monedas del sistema. Durante buena parte del período, la única moneda que fue objeto de crisis periódicas y frecuentes fue la libra esterlina, viéndose el gobierno británico obligado a adoptar lo que se denominó política de «stop and go», que alternaban períodos en que había que frenar el crecimiento para equilibrar su economía, con otros en que se relanzaba la economía, lo que llevaba, tras un cierto tiempo, a la reaparición del problema de la balanza de pagos. Éste obligaba de nuevo a frenar la economía, de tal forma que el ciclo, de pocos años de duración, volvía a empezar ${ }^{2}$.

A partir del otoño de 1967, la especulación dejó de estar centrada en la libra esterlina y comenzó a dirigirse directamente contra el dólar. A principios de 1968, tal especulación, actuando principalmente a través del mercado del oro en Londres, obligó a las autoridades monetarias de los principales países a abandonar su intento, iniciado en 1962, de estabilizar el precio en dicho mercado. Desde marzo de dicho año, el oro pasó a tener un doble precio: para las autoridades monetarias seguía valiendo 35 dólares la onza, el precio fijado por los Estados Unidos en los años treinta; para los particulares y empresas, el precio que alcanzaba en el mercado. Tan anómala situación pudo continuar varios años porque ambos conductos, el oficial y el privado, se mantuvieron separados. Para ello, las autoridades monetarias de los principales países establecieron un pacto comprometiéndose a no vender oro en el mercado privado. La contrapartida de ese acuerdo fue la inmovilización total del oro en las reservas de los países. Paralelamente se produjo un incremento de las presiones de los Estados Unidos sobre el resto de los países para que éstos no realizasen conversiones de dólares en oro.

Por este procedimiento se aisló al sistema de las presiones especulativas del mercado del oro, pero la especulación continuó intensamente en los mercados de divisas. Ello llevó a las devaluaciones del dólar en 1971 y 1973, hecho de particular relieve por tratarse de la moneda de reserva principal y casi única del sistema. La segunda de dichas devaluaciones no impidió que continuasen teniendo lugar fuertes movimientos especulativos, por lo que los Estados Unidos suprimieron formalmente la convertibilidad oro y establecieron la flotación de su moneda en marzo de 1973.

\footnotetext{
2 Un estudio más detallado del tema se encuentra en Varela Parache (1974).
} 


\subsection{El sistema de flotación y la intervención en el mercado de cambios}

El sistema de Bretton Woods desapareció bajo la presión de los hechos. Los bancos centrales y autoridades monetarias no deseaban el paso a la flotación; ésta se impuso, como situación de hecho, ante las presiones especulativas. De esta forma se produjo una divergencia entre la normativa sobre el sistema -el Convenio Constitutivo del FMI - y la realidad. La compleja situación internacional — primera subida del precio del petróleo, fuertes desequilibrios de las balanzas de pagos y desacuerdos en las políticas a aplicar - hará que esa situación se prolongue algunos años. Sólo concluirá con la aprobación de la Segunda Enmienda al Convenio Constitutivo, aprobada en 1976, que entró en vigor, tras las oportunas ratificaciones parlamentarias en 1978. Frente a las claras reglas precedentes, la nueva reglamentación es esencialmente permisiva. Simplemente indica que cualquier país puede establecer su tipo de cambio en relación con una moneda de otro país (cualquiera que ésta sea), con una moneda-cesto (media ponderada de monedas) de su libre elección o dejar que dichos tipos de cambio fluctúen en el mercado. Libertad completa para la elección del sistema que se complementa, además, por la posibilidad posterior de variar tal elección sin más requisito que comunicarlo al FMI en los treinta días siguientes. No resulta extraño que un sistema de estas características haya sido caracterizado por muchos autores como un «no-sistema» ${ }^{3}$.

La elección del sistema de fijación de los tipos de cambio por los países, llevó a que hubiera un predominio claro del número de países que eligieron la fijación del tipo de cambio en relación con una moneda nacional o un cesto de monedas. Pero la lista de países que escogieron la flotación de sus monedas comprendía los principales países industriales y representaba la gran mayoría de los movimientos comerciales y financieros del mundo. Por eso resulta correcto hablar del sistema practicado a partir de los años setenta como un sistema de flotación.

Dentro del nuevo sistema, los países podían haber elegido la opción de no intervenir en los mercados de cambio, dejando que éstos alcanzaran libremente su precio de equilibrio, como ocurre en cualquier otro mercado. Pero esto no fue así. Desde un principio, las autoridades monetarias consideraron el tipo de cambio como una variable demasiado importante para ser confiada exclusivamente a las fuerzas del mercado. Desde 1973, ha habido una flotación manejada por las autoridades que, en diversos momentos y con desigual frecuencia e intensidad, han tratado de influir sobre los tipos de cambio. Desde entonces se han producido variaciones importantes tanto en la naturaleza como en la forma de llevar a cabo las intervenciones.

${ }^{3}$ Véase Williamson y Milner (1991), pp. 387-390. 
En el primero de los aspectos, la intervención se realizó durante años respondiendo sólo a criterios nacionales. Cada gobierno decidía individualmente si intervenía o no y cuándo lo hacía. La experiencia derivada de esos años permitió distinguir entre paises que generalmente intervenían poco en los mercados de cambio, haciéndolo sólo ocasionalmente, como Estados Unidos; y países que lo hacían casi de forma cotidiana, como Francia o España. Las autoridades monetarias de cada país decidían la frecuencia y el volumen de la intervención y durante un periodo de doce años (1973-1985) lo hicieron en función de criterios estrictamente nacionales y de manera descoordinada. La flotación en este período se caracterizó por grandes vaivenes en los tipos de cambio, tanto nominales como reales, con largos períodos de apreciación o depreciación de las monedas. Concretamente la relación del dólar con las otras monedas principales del sistema se caracterizó por una importante depreciación del dólar hasta 1979 y una fuerte apreciación entre 1979 y 1985.

El año 1985 marca el principio de los intentos de intervención conjunta, por parte de los grandes paises, para tratar de influir coordinadamente en los mercados de cambios. En el origen de esta actuación internacional están las presiones proteccionistas ejercidas por importantes grupos industriales ante las dificultades competitivas derivadas del tipo de cambio del dólar, claramente sobrevaluado. Esto lleva al acuerdo, consagrado en la reunión del Plaza (Nueva York) en septiembre de 1985, por el que los principales países trataron de conseguir una caída paulatina del dólar que tuvo lugar en los años siguientes. Desde entonces se han producido intervenciones concertadas de distinto signo en diversos momentos, respondiendo siempre a decisiones tomadas en función de la evolución de los mercados y la conveniencia de intervenir conjuntamente para tratar de influir en ellos.

El otro factor, que ha jugado un importante papel en la evolución sistema, consiste en la sustitución de la intervención directa en el mercado de divisas por la indirecta a través de los tipos de interés. En un mundo con una creciente internacionalización de los flujos financieros, la intervención directa puede jugar sólo un papel modesto respecto a los volúmenes de divisas que se pueden mover en cualquier momento. Las posiciones que mantenían la ineficacia de las intervenciones en el mercado de divisas fueron ganando peso entre los economistas y alcanzaron máxima influencia en la primera mitad de los años ochenta en Estados Unidos, con el Gobierno de Reagan. Según la versión más radical, la intervención no tiene ningún efecto distinto del que pueden originar las contrapartidas monetarias que tal intervención origina. De acuerdo con este enfoque no hay política de intervención directa efectiva, sino efectos de la política monetaria sobre los tipos de cambio. En esa línea está también el Informe Jurgensen (1983) que señalaba que la intervención esterilizada - aquella cuyas contrapartidas monetarias internas se compensan con otras actuaciones monetarias de idéntica intensidad y distinto signo- no tenían un 
efecto substancial ni duradero en los tipos de cambio. Este enfoque se va a extender a otros países y determinar una menor intervención en el mercado de divisas y una sustitución por la utilización de los tipos de interés como mecanismo para influir en el equilibrio en el mercado de cambios. Es decir, la utilización de la política monetaria para este fin. Para algunos economistas ésta es la única forma de influir en el mercado de cambios; por el contrario, otros piensan que actuar directamente en el mercado de divisas puede representar un instrumento eficaz, al establecer la posición de las autoridades y su disposición a comprometer recursos para mantenerla ${ }^{4}$.

\subsection{Las esperanzas depositadas en los tipos flotantes y la realidad}

Muchas fueron las razones que apoyaban en los años setenta un sistema de libre flotación de las monedas y mucha la polémica que se ha generado sobre las diferencias entre las ventajas que este sistema ha proporcionado y las que se pronosticaron por sus partidarios.

Cuando se introdujeron los tipos de cambio flexibles en la década de los setenta, el panorama monetario mundial se caracterizaba por sucesivas crisis del sistema de paridades, por graves presiones especulativas sobre la moneda de reserva (el dólar) y por el temor de una propagación de la inflación estadounidense a otros países, como Alemania, facilitada por los tipos de cambio fijos. Por lo tanto, no es de extrañar que en aquel momento los tipos de cambio flotantes fuesen acogidos como una solución necesaria, ya que representaban, al menos teóricamente, el aislamiento nacional de las perturbaciones exteriores mediante las correspondientes variaciones del tipo de cambio (los países ya no se verían obligados a importar políticas externas indeseables). Al mismo tiempo, los tipos de cambio funcionarían como estabilizadores automáticos de la economía de los paises: su rápido ajuste aseguraría el equilibrio externo, y eliminaría los ataques especulativos al permitir la variación de los tipos de cambio nominales mantener constante el tipo de cambio real. Otra virtud atribuida a los tipos de cambio flexibles era liberar a los bancos centrales de la necesidad de intervenir en los mercados monetarios para mantener los tipos de cambio; con el nuevo sistema, el tipo de cambio sería un precio libremente fijado por las leyes del mercado, dando libertad a las autoridades monetarias para usar las medidas de política monetaria y fiscal para finalidades internas 5

+ Véase Domínguez y Frankel (1993).

" Para un análisis más completo de lo expuesto en este apartado puede consultarse Viñals (1986). El origen del debate sobre tipos de cambio flexibles puede hallarse en Friedman (1953) y en Meade (1955). Un análisis del cambio de enfoque posterior sobre el tema, en McKinnon (1981). 
Sin embargo, la realidad se ha ocupado de eliminar el aura con que se pretendía envolver a los tipos de cambio flotantes para demostrar que las grandes ventajas que se les atribuyeron sólo se han cumplido muy parcialmente, siendo muchos los objetivos que han quedado sin alcanzarse. Un breve repaso a lo acontecido en el panorama monetario internacional desde 1973 echa por tierra los argumentos mantenidos por sus defensores. ¿Por qué ha decepcionado el comportamiento de los tipos de cambio flotantes?

Básicamente se olvidó la idea de que no existe un sistema óptimo de tipo de cambio sino que depende, en cada momento, de la fuente y naturaleza de los shocks que sacuden las economías. Las perturbaciones de carácter monetario pueden ser amortiguadas a medio plazo por la flexibilidad de los tipos nominales, que se ajustan para mantener estable el tipo real; pero el sistema no permite el aislamiento a corto plazo de las economías nacionales. En el caso de perturbaciones reales, la actuación es aún más decepcionante, ya que no consiguen aislar las economías ni en el corto ni en el medio plazo, debido a que los tipos de cambio flexibles no pueden proporcionar un aislamiento efectivo contra cambios en los precios relativos entre diferentes clases de bienes (por ejemplo, entre el petróleo y los bienes de alimentación) porque no pueden modificar los precios relativos a ese nivel de desagregación. De hecho, los shocks más importantes de los años 70 fueron shocks reales que ocasionaron grandes cambios en los precios relativos de los bienes comercializables, sin que los tipos de cambio flotantes pudieran evitarlo ${ }^{6}$. Por lo tanto, aunque se había considerado a los tipos de cambio flotantes fundamentales para el aislamiento de las economías, esta capacidad de aislamiento se ha revelado como muy relativa, no pudiéndose generalizar sin «especificar la naturaleza de la perturbación (monetaria o real), el origen de la perturbación (interna o externa), qué es lo que se aísla (producto real o consumo) y quién va a ser aislado (país nacional o el país nacional y el extranjero en conjunto)»?

El papel de estabilizador automático que se le asignó tampoco se cumplió estrictamente. A corto plazo, los efectos de la «curva J» en la cuenta corriente eliminaban la automaticidad del ajuste que vendría a alcanzarse en el medio y largo plazo, siempre y cuando las características estructurales de la economía lo permitieran; en caso contrario, podía coexistir el equilibrio de la balanza de pagos con largos períodos de déficit corriente. Sin embargo, hay que reconocer que los tipos de cambio flotantes facilitan los ajustes de los tipos de cambio reales exigidos por cambios permanentes en los mercados de productos, por lo que ante los cambios estructurales experimentados por las economías mundiales desde los años setenta,

\footnotetext{
"Véase International Monetary Fund, Research Department (1984), p. 25.

Crockett y Goldstein (1987), p. 9.
} 
el sistema de tipos de cambio flexibles ha sido el mejor que se podía tener. Por último, respecto a la mayor libertad otorgada a las autoridades monetarias, al no tener que intervenir en los mercados de cambios, conviene señalar que éstas, muchas veces, no permanecieron impasibles a las variaciones del valor de su moneda. Como hemos visto, siguieron interviniendo en los mercados para el mantenimiento del tipo de cambio, tarea que se suponía no necesaria en un régimen de flexibilidad.

En resumen, la autonomía de la política monetaria permitida por el sistema de flotación no era tan grande como pretendieron sus defensores, ni era el sistema de flotación el mecanismo que independizara plenamente su actuación para la consecución de los objetivos internos. Sin embargo el fallo no estaba en el sistema sino en la naturaleza de la política en sí, que depende de los límites impuestos por el grado de apertura de las economías (la mayor conexión con los mercados internacionales obliga a llevar a cabo políticas monetarias condicionadas por las actuaciones exteriores). «Estos límites ponen de manifiesto una reducida capacidad para controlar los instrumentos de la política monetaria (la oferta de dinero nominal bajo tipos de cambio fijos) o una capacidad reducida para controlar algunos de los objetivos internos de la política monetaria (el nivel de producción real) o un incremento de la cautela en el uso de la política monetaria por sus efectos potencialmente peligrosos sobre las expectativas.» ${ }^{8}$

A pesar de haber incumplido la mayoría de las funciones que se le atribuyeron, la mayor crítica que se le puede hacer a los tipos de cambio flotantes no se basa en los argumentos anteriores sino en la extraordinaria volatilidad que han presentado en el día a día. Las voces de alarma ante la amplitud de las variaciones de los tipos de cambio comenzaron a escucharse a principios de los años ochenta. Las preocupaciones se agravaron ante la observación de la tendencia al desalineamiento de los valores de cambio de las monedas en periodos largos.

Si la variabilidad diaria del valor relativo de las monedas es un hecho grave «per se», en mayor medida lo será al venir acompañado de desviaciones entre el valor actual del tipo real de cambio y el tipo de equilibrio a largo plazo. La volatilidad del tipo de cambio nominal unida a la inflexibilidad de los precios de los bienes en el corto plazo tenía como resultado un ajuste excesivo del tipo de cambio real; como consecuencia la asignación de los recursos de la economía se producía ineficientemente ante la presencia de incertidumbre en la evolución de las tasas nominales y reales de cambio. Esta ineficiencia se reflejaba en el comercio y la inversión, elevando sus costes como consecuencia de la utilización de instrumentos de cobertura para eludir la inestabilidad del tipo de cambio.

* Véase Crockett y Goldstein (1987),p. 10. 
La volatilidad tiene su mayor aliado en la variación de las expectativas de los participantes en el mercado dada la elevada sensibilidad del tipo de cambio a las variaciones en las preferencias, a los cambios esperados de la política monetaria y a la credibilidad de dichas políticas. De lo incierto de las actuaciones de los agentes económicos se deriva lo incierto de la evolución esperada de la tasa cambiaria. Friedman no dijo que los tipos de cambio flexibles tenían que ser estables ${ }^{9}$; es más, dado su carácter de precio relativo, influido por los mercados de bienes y de capitales, podrían fluctuar volátilmente respondiendo a las variaciones en estos mercados. Sin embargo, aun conociendo la necesaria movilidad del tipo de cambio, lo preocupante es la magnitud que dicho vaivén adquirió en los mercados, muchas veces $\sin$ responder a factores reales. Este es el motivo principal que ha llevado a las propuestas realizadas en los últimos años para una mayor estabilización del sistema, imponiendo límites a la flexibilidad.

\section{PROPUESTAS PARA UNA MAYOR ESTABILIDAD DE LOS TIPOS DE CAMBIO}

El tiempo transcurrido desde que se inició la flotación de los tipos de cambios ofrece una experiencia no fácilmente conciliable con ninguna de las posturas extremas adoptadas antes de su establecimiento. No se han cumplido las expectativas negativas de quienes pensaban que la ausencia de tipos de cambio fijos significaría una caída del comercio y la inversión internacional y una mayor utilización del tipo de cambio como instrumento de protección. Pero todavía menos las de los defensores a ultranza de los tipos de cambio flexibles que esperaban un ajuste automático de las balanzas de pagos, dejando libre el instrumento de la política monetaria para conseguir objetivos internos. Como hemos señalado, los tipos de cambio de las principales monedas han mostrado grandes oscilaciones, tendiendo a realizar fluctuaciones amplias en torno al nivel de equilibrio, más que acercarse rápidamente a él. Como ha señalado Williamson ${ }^{10}$, los años transcurridos han mostrado que los tipos de cambio flexibles son viables pero no existe consenso sobre que tal sistema sea deseable.

No resulta extraño que ante la fuerte volatilidad y las grandes oscilaciones entre los tipos de cambio de las principales monedas, registrados desde los años setenta, hayan surgido propuestas orientadas a limitar tales fluctuaciones, buscando una mayor estabilidad de tipos de cambio. Tampoco que desde posturas opues-

\footnotetext{
`Véase Meltzer (1993), p. 115.
}

10 Véase Williamson (1983), p. 9. 
tas se considere que el sistema de flotación ha tenido que soportar muy fuertes perturbaciones que hubiesen acabado con cualquier otro sistema alternativo, salvo que se hubiesen establecido estrictos controles de capital ${ }^{11}$. Menos todavía puede extrañar que la controversia entre ambas posturas haya estado entretejida con la evolución de la experiencia europea en su intento de limitar y, en el futuro, eliminar la fluctuación entre monedas comunitarias.

Nos referimos en este apartado a determinadas propuestas de estabilización de los tipos de cambio, en particular la realizadas por Williamson y McKinnon, las más importantes que se han elaborado en respuesta a las fuertes fluctuaciones de los tipos de cambio en los años setenta. Ambas son teorías que pretenden un sistema de tipos de cambio controlado internacionalmente por los principales países, con objeto de limitar las fluctuaciones de los tipos de cambio; pero emplean mecanismos muy diferentes para conseguirlo. Añadiremos a ello la propuesta de Tobin de limitar la libertad de los movimientos de capital; propuesta que ha adquirido una particular actualidad tras el protagonismo adquirido por éstos en la reciente crisis monetaria de los países europeos.

\subsection{Propuesta de las «zonas objetivo»}

Fue inicialmente realizada por Bergsten y Williamson (1983), posteriormente desarrollada por Williamson (1983) y refinada y ampliada por Williamson y Miller $(1987)^{12}$. La propuesta parte de que cualquier país trata de conciliar tres objetivos macroeconómicos: nivel de actividad elevado, estabilidad monetaria y balanza corriente equilibrada o con déficit financieramente sostenible. Cada país, individualmente, puede buscar una composición distinta de esos tres objetivos parcialmente contradictorios. El objetivo de la propuesta es buscar un mecanismo internacional capaz de compaginar el equilibrio interno y externo de los principales países.

Para ello, Williamson introduce el concepto de «tipo de cambio de equilibrio fundamental» (TCEF), definido en sentido implícito en el Convenio Constitutivo del Fondo Monetario Internacional. Éste hace referencia al desequilibrio fundamental como aquel que no puede corregirse mediante mecanismos internos sin un alto coste en términos de crecimiento y empleo, por lo que se justifica el ajuste de la

11 Véase Root (1994), p. 507.

12 El enfoque ha dado lugar en los últimos años a modelos más formalizados del tipo de cambio que parten de Krugman (1991) y tratan de evitar los supuestos de partida de este autor (perfecta credibilidad de la zona objetivo e inexistencia de intervenciones intramarginales), claramente rechazados empíricamente. Véase Garber y Svensson (1994). 
paridad. En la propuesta de Williamson, los países tendrían como objetivos mantener los tipos de cambio dentro de unos márgenes relativamente amplios en torno al TCEF, el cual iría cambiando a medida que variasen las condiciones básicas de la economía. De esta forma, la propuesta de las «zonas-objetivo» tiene algo en común con los dos principales sistemas de flexibilidad de los tipos de cambio propuestos en los años sesenta: en paralelismo con el ajuste paulatino de paridades, también hay un ajuste en el campo de variación posible de los tipos de cambio; pero los márgenes de fluctuación a corto plazo son amplios, todavía en mayor medida de lo propugnado entonces por los partidarios del ensanchamiento de las bandas de fluctuación.

El concepto de TCEF y su ajuste parte de la distinción $-\mathrm{y}$ distinta valoración- que Williamson hace de volatilidad y desalineamiento de los tipos de cambio. La volatilidad es un concepto a corto plazo y mide la variación que ha tenido el tipo de cambio durante un período dado. Por el contrario, el desalineamiento trata de medir la separación del tipo de cambio de su nivel de equilibrio a largo plazo, representado por el TCEF. Para el autor, el desalineamiento de los tipos de cambio tiene mucha más trascendencia económica que su volatilidad, cuyos efectos negativos pueden ser contrarrestados por el desarrollo de mercados a plazo y otros mecanismos de cobertura. El TCEF puede cambiar en el curso del tiempo - diferente ritmo de inflación o variaciones en la demanda real o en la capacidad de financiación de una economía - pero no es probable que cambie frecuente y drásticamente. Bastará hacer su modificación en momentos discontinuos, con una cierta periodicidad. Para ello, la institución rectora -el FMI- tendría como misión seguir la evolución de las variables que influyen en el TCEF de cada país, sirviendo estos cálculos para establecer periódicamente las nuevas bandas de fluctuación; es decir, las nuevas «zonas objetivo».

El compromiso de los países consistiría en mantener el tipo de cambio de sus monedas respectivas dentro del campo de la variación de esas «zonas-objetivo» que irían cambiando periódicamente al hacerlo las condiciones básicas de la economía. Pero ni siquiera este sería un compromiso rígido. Aunque las «zonasobjetivo» serían publicadas y conocidas por todos, sus límites no serían estrictos sino que podrían ser temporalmente sobrepasados; habría márgenes blandos, más que una obligación exacta de mantener los tipos de cambio dentro de la zona.

Además del mencionado objetivo exterior, los países se comprometerían también a conseguir un objetivo de carácter interno: un cierto crecimiento de la demanda interna nominal. Su propósito es poner un límite al crecimiento de la inflación. Para ello se utiliza como variable intermedia no la renta nacional sino la demanda interna porque de esta manera un país con elevado déficit por cuenta 
corriente tendrá que mantener un crecimiento de la renta, menor que el deseado para conseguir el equilibrio global; por el contrario, el país con superávit de balanza corriente podrá conseguir un crecimiento por encima del crecimiento global al sumarse a su demanda interna la externa.

Para trasladar esos dos objetivos interior y exterior en reglas de actuación política, Williamson establece tres reglas básicas:

1." El diferencial de los tipos de interés debe emplearse prioritariamente para mantener los tipos de cambio dentro de la «zona-objetivo». Para Williamson los tipos de interés son el instrumento más eficaz para intervenir en el mercado de cambios. Pero su empleo prioritario para este fin no quiere decir empleo exclusivo: la amplitud de la banda de fluctuación permite que también puedan emplearse para conseguir objetivos internos, salvo en situaciones muy especulativas.

2." Suponiendo que el diferencial de los tipos de interés entre las dos monedas no es el adecuado para conseguir mantener los tipos de cambio dentro de las «zonas-objetivo», habrá que determinar cuál de los dos países le corresponde realizar el ajuste. Williamson lo hace depender del crecimiento de la demanda agregada global. Si ésta es mayor de lo deseable, el país con situación débil de balanza de pagos debe incrementar sus tipos de interés; en el caso contrario, el país con superávit deberá reducir el suyo. Esta regla trata de conseguir la simetría en el ajuste, atribuyendo a uno u otro país o grupo de países la obligación de realizarlo.

3. a Cuando la política monetaria no consiga los niveles pretendidos de demanda interna, puede ser necesario una utilización keynesiana de la política fiscal para conseguir tal propósito ${ }^{13}$.

Hemos hecho una amplia referencia a la propuesta de las «zonas-objetivo» porque es, sin duda, la propuesta que ha tenido más amplia repercusión en la literatura económica, ha sido objeto de tratamiento en el FMI ${ }^{14}$ y recientemente fue apoyada por el Informe de la llamada Comisión de Bretton Woods. Para sus partidarios la propuesta mejoraría claramente el «no sistema» actual, permitiendo, sin embargo, suficiente flexibilidad para evitar crisis especulativas como las experimentadas por el Sistema Monetario Europeo (SME). Las críticas a la propuesta de las «zonas

13 Véase Williamson y Miller (1987) y Williamson (1990) para una exposición más amplia de esta propuesta y otros puntos tratados en la exposición.

14 Véase Crockett y Goldstein (1987). 
objetivo» se han centrado, por una parte, en la dificultad de su definición y de la medida de sus variaciones; por otra, en la exigencia de un esquema de cooperación difícilmente alcanzable —como el antes descrito- si no se quiere que las políticas puedan tener efectos perturbadores ${ }^{15}$. El problema principal del sistema es que su implantación exigiría una pérdida de independencia en la utilización de la política monetaria para fines internos. La importancia de dicha pérdida depende, por una parte, de los supuestos a los que se ajuste el modelo. Si las zonas establecidas son creíbles, como supone Krugman (1991), cuanto más se aproxima el tipo de cambio al extremo de fluctuación, menor es la necesidad de adoptar medidas monetarias que podrían ser indeseables desde un punto de vista interno. Pero la experiencia empírica, corroborada por la crisis monetaria europea a principios de los años noventa, parece distanciarse de dicho supuesto; más bien apunta a la falta de credibilidad en los límites de fluctuación, incluso en casos de países que mantienen los equilibrios básicos de su economía. De esta manera, el sacrificio de la política monetaria a la consecución del objetivo externo es importante. El otro factor que puede hacer la pérdida de independencia monetaria menos relevante es que los países estén sometidos a «shocks» de carácter simétrico, ya que, entonces, las medidas que pueden tomarse por la autoridad monetaria - sea ésta de un país individual o un banco central conjunto- serán igualmente acertadas —o desacertadas- para los demás.

Parece evidente que este no es el caso de los tres países cuya moneda constituye el núcleo principal del actual sistema monetario internacional. Los tres países han dado muestras evidentes de que consideran fundamental retener la independencia de su política monetaria, de forma que pueda emplearse primordialmente para conseguir el equilibrio interno. Estos países consideran indeseable, tanto individual como globalmente, sacrificar la estabilidad económica interna a la consecución de una mayor estabilidad de los tipos de cambio y lo consideran inadecuado no sólo a efectos internos sino también de la economía mundial en su conjunto. Por ello, se estima que «una reforma fundamental del presente sistema monetario internacional para transformarlo en un sistema de zonas objetivo no es ni deseable ni posible» ${ }^{16}$.

15 Williamson y Milner (1991) se refieren a este aspecto en relación con la política de Estados Unidos, a principios de los años ochenta, para limitar la apreciación del dólar. Se dice que - de acuerdo con la propuesta - las autoridades deberían haber suavizado la política monetaria a principios de los años ochenta, lo cual hubiese llevado a una mayor inflación. Sin embargo, los autores señalan que tal situación también hubiera llevado a llamar la atención sobre la inconsistencia de la política fiscal establecida. Para una crítica y análisis detallado de los principales aspectos de la propuesta Williamson, véase Frenkel y Goldstein (1986).

it Véase Mussa et al (1994), p. 32. 


\subsection{Propuesta de un crecimiento monetario global}

McKinnon (1988) considera indudable la conveniencia de los tipos de cambio fijos que, en su opinión, vienen avalados por el mejor funcionamiento de la economía mundial durante los períodos en que predominó tal sistema. Pero, además, señala que el recurso a los tipos de cambio flexibles como mecanismo de ajuste ha perdido su eficacia con el aumento de la interdependencia económica. La doctrina económica del enfoque de las elasticidades de Robinson (1937) o Meade (1951), en virtud de la cual una modificación súbita del tipo de cambio mejora la balanza de pagos, podía ser en buena parte cierta en los años treinta o cincuenta, cuando ambos la expusieron. Pero en el mundo actual, los tipos de cambio no tienen un efecto tan claro a causa de sus repercusiones macroeconómicas más amplias. Las variaciones del tipo de cambio las determina no tanto el flujo de bienes y servicios - balanza corriente- como las expectativas de los agentes económicos sobre el valor futuro de los activos en distintas monedas. Así, el mercado de cambios de las monedas en régimen de flotación se ajusta pasivamente a las volátiles preferencias de los tenedores de activos ${ }^{17}$.

Para McKinnon (1988) la volatilidad de los tipos de cambio es el resultado del carácter sustitutivo de las monedas entre sí, dada la libertad creciente de los movimientos de capital, argumentación que ha ganado peso, indudablemente, con la creciente libertad e internacionalización de los movimientos de capital que ha caracterizado a los últimos años. Las grandes instituciones de inversión internacional y los inversionistas en general, mantienen sus fondos en diversas divisas. El volumen total invertido puede experimentar variaciones, pero este cambio es pequeño y paulatino. Los cambios importantes a corto plazo, sólo se producirán en la composición de las carteras. En un determinado momento puede haber un mayor deseo de mantener dólares que marcos y los cambios en la composición global de las carteras originará las correspondientes presiones en el mercado de cambios.

A partir de esta idea McKinnon (1988) planifica lo que él llama un «patrón oro sin oro». Debe haber un crecimiento de la liquidez global que se establezca conjuntamente por los países, sustituyendo a la práctica actual, en virtud de la cual cada país establece su propio objetivo de crecimiento de las magnitudes monetarias. De acuerdo con su propuesta para la existencia de un sistema monetario internacional ordenado, bastaría que los tres países cuyas monedas juegan un papel clave actualmente (Estados Unidos, Alemania y Japón) se pusieran de acuerdo en mantener un ritmo determinado de expansión monetaria global y en fijar definitivamente los tipos de cambio entre ellos, sin esterilización alguna de los efectos mone-

17 Véase McKinnon (1988), p. 94. 
tarios de cualquier desequilibrio de balanza de pagos que se pueda producir. De esta forma, un aumento de la preferencia por el marco en el conjunto de las carteras internacionales, determinará un aumento de la circulación en marcos y un decrecimiento de la circulación en dólares. El incremento de la liquidez global acordado no variará, pero sí la distribución entre los distintos mercados. La propuesta de McKinnon responde a una especie de monetarismo global, ya que se produciría un incremento automático de la liquidez conjunta, cuya distribución entre monedas se vería afectada exclusivamente por la voluntad de los inversionistas. Todo ello significaría una renuncia total a emplear la política monetaria para finalidades internas. De esta forma, según la propuesta de McKinnon los bancos centrales se limitarían a establecer un nivel de tipos de cambio nominales con bandas de fluctuación estrechas. El tipo de cambio fijado ya no tendría que ser alterado. Todo el ajuste necesario se produciría a través de los movimientos de oferta monetaria que determinarían el adecuado crecimiento de la inflación. Implícito está en su argumentación el cumplimiento de la teoría cuantitativa y de la neutralidad del dinero; también el perfecto funcionamiento de los mercados y la no existencia de presiones especulativas, al aceptar el público la permanencia de los tipos de cambio fijos ${ }^{18}$. Se trata de una posición monetarista extrema «al negar la importancia de la política fiscal y la necesidad de utilizar la política monetaria para cualquier propósito de equilibrio interno distinto de conseguir la estabilidad secular de los precios de bienes objeto de comercio» ${ }^{19}$. Todo ello ha hecho extremadamente difícil la aceptación de esta propuesta como base de actuación práctica para conseguir una mayor estabilidad de los tipos de cambio.

\subsection{Propuesta de control de los flujos especulativos}

Tobin es uno de los primeros autores que han llamado la atención sobre el efecto perturbador que pueden tener los crecientes flujos privados de capital a corto plazo sobre los tipos de cambio. En un trabajo publicado en 1978 señalaba que los gobiernos, en muchos casos, no pueden absorber los importantes flujos internacionales de capital sin sacrificar sus objetivos internos en términos de empleo, producción e inflación porque «la movilidad de los capitales financieros limita las diferencias entre los tipos de interés nacional y esto restringe fuertemente la capacidad de los gobiernos y bancos centrales para proseguir las políticas monetarias y fiscales adecuadas para su economía internas ${ }^{20}$.

\footnotetext{
${ }^{18}$ Davidson (1992-93), pp. 168 y ss.

14 Véase Williamson y Milner (1991).

${ }^{20}$ Véase Tobin (1978), p. 154.
} 
Para evitar estos efectos indeseados, especialmente patentes en momentos de ataque especulativo, Tobin proponía la introducción de un impuesto que gravara a todas las operaciones con objeto de que los mercados de cambio no tuviesen tanta fluidez y los especuladores encontraran menos rentable las rápidas operaciones de entrada y salida en cualquier moneda. Dicho impuesto se deberia establecer a un nivel relativamente bajo, como un $1 \%$, de tal forma que no significara un gravamen importante sobre las operaciones de carácter permanente, pero sí sobre las de carácter especulativo, por definición a muy corto plazo. Tobin reconoce el efecto perjudicial que un impuesto de esta naturaleza tendría para el comercio internacional, pero estima que globalmente la economía saldría ganando al verse menos sometida a estas perturbaciones exteriores.

La propuesta de Tobin pretendía simplemente reducir la volatilidad del sistema de tipos de cambio flotantes. Perdió vigencia en los años ochenta como consecuencia de las posturas en favor de la libertad de funcionamiento de los mercados que los han caracterizado: la intervención de las autoridades parecía no sólo muy difícil de realizar, sino sencillamente indeseable. Era citada por aquellos que se preocupaban de los efectos de la movilidad de capital pero no compartida por la mayoría de ellos. Recientemente, sin embargo, le ha dado una nueva actualidad la crisis monetaria de 1992-93 y el efecto que en ella ha jugado la liberalización de capitales. En los ataques especulativos registrados en dicho período, la mayor parte se pueden explicar en función de desequilibrios básicos de la economía, pero otros parecen responder al modelo elaborado por Obstfeld (1986) en el que las expectativas de devaluación son las que generan la propia devaluación, al originar fuertes flujos especulativos que producen tal resultado ${ }^{21}$. La propuesta de limitar los movimientos especulativos realizada por Eichengreen y Wyplosz (1993) y su refrendo y mayor desarrollo realizado por los mismos autores y Tobin (en un artículo posterior de 1995) ha contribuido a darle una nueva actualidad. El sistema propuesto en el primero de los trabajos partía de las medidas específicas adoptadas por el Banco de España con ocasión de la crisis, en el verano de $1992^{22}$; medidas que si bien tuvieron cierto impacto a corto plazo, la experiencia ha demostrado que su eficacia disminuyó después, al mismo tiempo que plantearon no pocos problemas al sistema financiero y afectaron no sólo a los flujos especulativos sino también a los movimientos de capital permanente. Pese a reconocer ese carácter transitorio y la imposibilidad de aislar totalmente los efectos antiespeculativos pretendidos, Eichengreen, Tobin y Wyplosz (1995) han insistido en la conveniencia de los con-

${ }^{21}$ Véase Santillán (1994), que constituye un excelente tratamiento en español del papel de las restricciones de capital.

${ }^{22}$ Sobre ello puede consultarse Linde (1993) y Alonso y Linde (1993). 
troles de capital como único método de hacer compatible la elevada movilidad de capitales del mundo actual con un sistema de tipos de cambio fijos pero ajustables, como todavía pretende serlo el Sistema Monetario Europeo. Para ello estudian la doble alternativa de un impuesto global sobre las transacciones y el de un impuesto sobre los préstamos a no-residentes. Pese a admitir el coste que significan tales medidas y su efecto temporal, los autores defienden la conveniencia de «echar arena en las ruedas» — tal como Tobin (1978) había descrito el proceso- y así hacer compatible los movimientos de capital con una «modesta autonomía nacional de política monetaria y macroeconómica» ${ }^{23}$. Otros, sin embargo, ven tales medidas como de imposible aplicación ${ }^{2+} \mathrm{e}$ implicando costes intolerables para el funcionamiento de los mercados de cambios y los mercados financieros ${ }^{25}$.

Dos observaciones conviene destacar de lo anteriormente tratado. La primera es que, aunque se habla de controles de capital, en la actualidad nadie pretende volver a controles tradicionales, en forma de prohibición, necesidad de autorización, etc. Se trata simplemente de hacer más caras o más difíciles las operaciones especulativas a corto plazo y el problema es si ello puede llevarse a cabo y con qué costes. La segunda es que todos los trabajos recientes mencionados están centrados en el Sistema Monetario Europeo y su situación presente (la segunda etapa del proceso evolutivo creado en Maastricht). Una aplicación en el contexto global parece difícilmente realizable.

\section{LA LIBERALIZACIÓN DE LOS MOVIMIENTOS DE CAPITAL Y LA EXPERIENCIA EUROPEA}

El desencanto con los tipos de cambio flexibles ha llevado a formular propuestas, como las mencionadas, que tienen por objeto conseguir un sistema con mayor estabilidad en los mercados de cambios. Sin embargo, paralelamente se ha producido una rápida internacionalización de los mercados de capitales que hace más difícil lograr la estabilidad pretendida. Tal internacionalización ha afectado incluso a mercados de capital que hace pocos años eran sólo mercados puramente domésticos. Esto ha hecho que los flujos de capital tengan una influencia decisiva en la determinación del equilibrio en los mercados de cambio y en los mercados nacionales de obligaciones, vehículo principal de la inversión internacional. La crisis monetaria europea ha puesto de relieve la incidencia de los movimientos de

23 Véase Eichengreen et al. (1995), p. 163.

${ }_{24}$ Véase Garber y Taylor (1945).

25 Véase Kenen (1995), p. 181. 
capital sobre los tipos de cambio. En particular ha arrojado nueva luz sobre la dificultad de conciliar un sistema de tipos de cambio fijo con la libertad de los movimientos de capital, a menos que los países estén dispuestos a renunciar a la independencia de su política monetaria. Por ello dedicaremos este epígrafe a describir la rápida expansión de los mercados financieros internacionales en los últimos años y su incidencia específica en la crisis europea, lo que nos permitirá sacar conclusiones sobre la evolución previsible del Sistema Monetario Internacional.

\subsection{Internacionalización de los mercados financieros}

La fuerte expansión de los movimientos de capital es una de las características de la evolución registrada durante los últimos quince años. En los paises industriales ha habido un proceso cada vez más amplio de liberalización de los movimientos de capital que, en años recientes no sólo ha ganado en profundidad sino que se ha extendido a un número apreciable de paises en desarrollo. La liberalización creciente de los movimientos de capital se ha conjugado con una serie de factores técnicos e innovaciones financieras, originando una expansión sin precedentes de las operaciones de capital transfronterizas, especialmente notoria a principios de los años noventa.

En la evolución que han experimentado los mercados financieros, la OCDE ha señalado que se pueden distinguir dos períodos distintos. Antes de la segunda mitad de los años ochenta, la internacionalización de los mercados se produjo principalmente mediante los mercados «off-shore»: primero a través del mercado de eurodivisas; después, especialmente en los años ochenta, mediante el desarrollo de euromercados sin intermediación bancaria (Eurobonos, Europapel comercial, etc...). Pero la segunda mitad de los ochenta y el principio de la actual década se han caracterizado por el desarrollo de la financiación a través de los mercados nacionales, utilizando principalmente el vehículo de la deuda pública. Hay una internacionalización sin precedentes de los mercados financieros hasta entonces típicamente nacionales.

Esta creciente internacionalización de los mercados nacionales ha ido acompañada de otro cambio estructural que lo favorece: el crecimiento de la inversión institucional. En todos los mercados financieros ha habido una concentración de la inversión en grandes entidades institucionales especializadas. Entidades que están en mejores condiciones que cualquier otro inversionista, para afrontar los problemas que necesariamente implican las inversiones transfronterizas.

Cálculos oficiales a principios de los años noventa cifraban en 2,5 billones de dólares las inversiones de esta naturaleza, cifra que se repartiría aproximadamente 
por igual entre acciones y otros títulos. Toda esta internacionalización e institucionalización de las carteras ha ido acompañado de un crecimiento espectacular de las operaciones de cambio ligadas con ellas. Hace tiempo que se viene manejando la cifra de un billón de dólares de movimientos diarios en los mercados de cambios, de los cuales sólo una pequeña proporción puede corresponder a pagos por bienes y servicios, constituyendo el resto transacciones financieras. Por tanto, el espectacular crecimiento del mercado de cambios entronca directamente con la expansión e internacionalización de las operaciones de capital. Con flujos de tal magnitud es fácil suponer su influencia decisiva en los tipos de cambio. También que las autoridades monetarias nacionales hayan ido complementando cada vez $-\mathrm{y}$, en muchos momentos, sustituyendo- sus intervenciones en el mercado de cambios por actuaciones de politica monetaria que varían el atractivo relativo de los activos nacionales respecto a los activos extranjeros. Por último, tampoco puede extrañar que las variaciones bruscas en los flujos de fondos originen crisis importantes en los mercados. A este respecto, lo ocurrido en Europa en el periodo 1992-93 es significativo y permite sacar enseñanzas válidas para establecer las conclusiones de este trabajo.

\subsection{La experiencia del Sistema Monetario Europeo (SME) ${ }^{26}$}

La difícil coexistencia entre la liberalización de los movimientos de capital y los ajustes esporádicos de los tipos de cambio ya fue apreciada, como hemos visto, en la época de Bretton Woods, pese a la liberalización sólo parcial de tales movimientos y a que todavía estaba lejos la explosiva internacionalización de las carteras a la que nos hemos referido en el epígrafe anterior. El tema ha sido objeto de amplia atención como consecuencia de la crisis monetaria en Europa durante los años 1992-93, en la que incidieron la plena liberalización de capitales, en un contexto financiero cada vez más globalizado, con la rigidez creciente de los tipos de cambio dentro del SME.

Una de las primeras llamadas de atención sobre esta inconsistencia fue la de Wyplosz (1988), que destacó la importancia que los controles de capital habían tenido hasta entonces para los participantes en el SME. Dichos controles permiten una cierta desconexión temporal de una economía respecto a los mercados internacionales. No son útiles durante largos períodos, porque siempre existen métodos de eludirlos. Ni pueden evitar las pérdidas de reservas o las depreciaciones si se sigue

\footnotetext{
26 En este epígrafe se utiliza ampliamente Varela (1994b).
} 
una política monetaria que sistemáticamente acomoda la inflación ${ }^{27}$. Pero son útiles durante períodos de crisis, si éstas no duran mucho. Actúan limitando la cuantía de los fondos especulativos que se pueden mover en un determinado momento.

Diversos autores han subrayado la contradicción que implica el período transitorio de la UEM, al coincidir la plena liberalización de los movinientos de capital y la inmovilidad de los tipos de cambio, necesaria para cumplir las condiciones de convergencia. Padoa-Schioppa ${ }^{28}$ ha señalado que, desaparecido el control de capitales, en las economías de los países comunitarios aparece lo que él denomina el «cuarteto inconsistente», ya que liberalización comercial, plena libertad de capitales, tipos de cambio fijos y autonomía de la política monetaria resultan incompatibles. Para preservar el mantenimiento inamovible de los tipos de cambio hará falta renunciar a la autonomía de la política monetaria o a la plena libertad de movimientos de capitales.

$\mathrm{Al}$ eliminarse los controles de capital en un mundo financiero crecientemente globalizado, las autoridades monetarias encuentran que las reservas son insuficientes para hacer frente a posibles movimientos especulativos y que éstos sólo pueden contrarrestarse mediante un aumento significativo de los tipos de interés. Tal aumento plantea muchos problemas, especialmente si las crisis son duraderas. Por ello, la ausencia de controles de capital ha cambiado radicalmente las condiciones en las que se desenvuelve la política monetaria en los países de la Unión Europea ${ }^{29}$.

Conviene subrayar que la incompatibilidad de las políticas mencionadas es una posibilidad, pero no una necesidad. Buena prueba de ello es el período que media desde que se empieza a avanzar en el proceso de creación de la UEM y el verano de 1992. Durante dicho período, el convencimiento de los agentes económicos sobre la estabilidad de las monedas comunitarias y el avance de la convergencia, condujeron a un apreciable desplazamiento de capitales hacia países con elevado déficit corriente, como España e Italia. Sus monedas se situaron en la parte alta de las bandas de fluctuación y se redujeron los diferenciales de sus tipos de interés con el marco. Los movimientos de capital jugaron un papel «estabilizador», aunque ya entonces surgieron algunas voces discrepantes, incluyendo a dirigentes del Bundesbank, sobre la conveniencia y el carácter auténticamente estabilizador de tales flujos. Pero bastó que a los desequilibrios y divergencias económicas se unieran unos cuantos hechos negativos sobre la evolución esperada de la Unión Económica y Monetaria para que el SME sufriera la mayor crisis de su existencia; una crisis cualitativamente diferente de otras anteriores.

\footnotetext{
27 Véase Smaghi y Micossi (1990), p. 149.

2* Véase Padoa-Schioppa (1988), pp. 372 y ss.

${ }^{24}$ Véase Eichengreen y Wyplosz (1993), pp. 62-63.
} 
De la experiencia histórica se extrae la constatación de que las expectativas sobre cambios en las políticas fijadas pueden producir apreciables flujos financieros que contribuirán al desequilibrio. Esta enseñanza se confirmaba de forma extrema con la plena movilidad de capitales establecida en los países de la Unión Europea a partir de 1990. Probablemente, razones de fondo no faltaban. Por una parte, la evolución de los costes y precios había sido muy distinta en los diferentes países participantes en el SME; esto tenía antecedentes, pero mientras en el pasado el efecto de tal divergencia en la competitividad se había visto compensado regularmente por movimientos de la paridad central, desde 1987 no había tenido lugar ningún ajuste de los tipos de cambio. Por otra parte, desde principios de los años noventa el sistema tenía un nuevo factor de inestabilidad que afectaba al país en que el SME descansa. A causa de la Reunificación, Alemania tuvo que incurrir en un déficit fiscal sin precedentes que llevó al Bundesbank a introducir una política monetaria tensa que, a medida que transcurría el tiempo, se hizo cada vez más conflictiva e indeseable para la mayoría del resto de los países europeos. No le ocurría igual a aquel país que había entrado más tarde en la depresión y cuyas autoridades daban carácter prioritario a la necesidad de controlar las presiones inflacionistas.

Pese a que esas discrepancias se fueron haciendo cada vez más patentes, los flujos de capital jugaron durante un período apreciablemente largo el papel «estabilizador» a que se ha hecho referencia. Pero lo que a fines de los ochenta y principios de los noventa fueron movimientos de capital equilibradores, iban a convertirse a partir de 1992 en desequilibradores: bastaría que al mencionado conflicto entre los objetivos internos y externos se uniese a un factor aparentemente anecdótico, como fue el resultado negativo del referéndum danés, para introducir serias dudas respecto a las perspectivas futuras de integración monetaria en Europa.

La fragilidad que estaba demostrando el proyecto de Unión Económica y Monetaria puso de relieve la potencialidad especulativa y en este caso, «desestabilizadora», de los mercados financieros. Es bien conocida la evolución registrada a partir del verano de 1992: ataques especulativos, inicialmente centrados en la libra y la lira, que llevaron a la salida de ambas monedas del mecanismo de tipos de cambio del SME; posteriores ataques a otras muchas monedas, algunas de las cuales, como la peseta, el escudo, o la libra irlandesa, sufrieron devaluaciones en más de una ocasión; y continuos ataques de gran envergadura al franco francés que sólo sale de tal trance con el masivo apoyo de Alemania. Pese a la intervención en montos sin precedentes de las autoridades monetarias europeas, fue necesario, finalmente, ampliar los márgenes de fluctuación hasta un $15 \%$, disfrazando una entrada encubierta de la flexibilidad de los tipos de cambio dentro del SME. 
Desde la ampliación de las bandas de fluctuación, el SME ha vivido un período de mayor calma, alterado en los primeros meses de 1995. La crisis del peso mejicano y su efecto sobre el dólar, han determinado presiones alcistas sobre el yen y el marco. Como consecuencia del fenómeno de polarización dólar-marco, hubo unas presiones al alza de esta moneda respecto a otras monedas europeas, originando apreciable tensión en los mercados de cambios. En todo caso, la situación se saldó con crisis menos dramáticas que en 1992-93. En un sistema con tipos de cambio fijos los especuladores juegan con ventaja porque obtendrán grandes beneficios si la operación realizada tiene éxito, mientras que, en caso contrario los costes son irrelevantes. Ello contribuye a hacer difícilmente conciliables la libertad de los movimientos de capital con el mantenimiento de los tipos de cambio fijos, a menos que los países renuncien total e irrevocablemente a la utilización de la política monetaria para fines distintos de conseguir el equilibrio exterior. La ampliación generosa de los márgenes de fluctuación hasta el 15\%, permitió el ajuste necesario y la crisis de principios de 1995 pudo saldarse sólo con la devaluación de la peseta y la refleja del escudo portugués.

Las consecuencias de la crisis monetaria europea proporcionan una valiosa experiencia para el sistema monetario internacional. Cualquier modificación de éste ha de partir de la rápida expansión y globalización de los mercados monetarios y de la dificultad de conciliar tal desarrollo con un sistema de tipos de cambio fijos si antes los países no han renunciado a la autonomía de sus políticas monetarias. Algo no fácil en el contexto europeo y difícilmente imaginable en el mundial.

\section{CONCLUSIONES}

La introducción de los tipos de cambio flotantes significó un cambio de gran trascendencia en el sistema monetario internacional. Aunque la modificación se produjo exigida por las circunstancias -especulación sobre el dólar-, muchos economistas consideraron que el nuevo sistema, con flexibilidad de los tipos de cambio, constituía un objetivo deseable en sí mismo. Sin embargo, los resultados de tal sistema, en términos de estabilidad de los mercados y de independencia de la política monetaria de los países, han estado por debajo de lo esperado por sus partidarios. Ante fuertes fluctuaciones de los tipos de cambio no es extraño que se hayan buscado fórmulas para tratar de conseguir una mayor estabilidad de los mercados de cambios.

La experiencia de los últimos años parece arrojar resultados contradictorios sobre la controversia de si es conveniente o no un sistema más estable de tipos de cambio. Las grandes fluctuaciones de los tipos de cambio de las principales mone- 
das de los años setenta y ochenta y los progresos de convergencia entre los países europeos logrados durante la parte final de los ochenta, parecían inclinar la balanza hacia mecanismos de mayor estabilidad que encontraban en el SME su paradigma. Pero la evolución reciente ha representado un retroceso en las posturas favorables a una mayor estabilidad.

Por una parte, el proceso de globalización financiera ha tomado a partir de los años ochenta, especialmente en su segunda mitad, nuevos impulsos, al tiempo que tenía lugar un cambio cualitativo en su forma de actuar, incidiendo directamente sobre los mercados nacionales, y de forma particular en los de deuda pública. Aunque los mercados financieros distan de alcanzar un auténtico carácter global, su internacionalización ha aumentado notablemente y afecta a mercados nacionales de países que hace unos años hubiera sido impensable que formaran parte de las alternativas de inversión de las grandes entidades financieras. Las decisiones de inversión de estas últimas influyen en los mercados de divisas y de obligaciones de los países, haciendo de los movimientos de capital un factor clave para la determinación de su equilibrio.

Cualquier modificación que en el futuro pretenda introducirse en el sistema monetario internacional tiene que tener en cuenta esa realidad de la movilidad de los capitales, así como la experiencia acumulada en la crisis de 1992-93 del SME. Ésta nos indica que los capitales pueden jugar un papel equilibrador y moverse en cuantías importantes hacia países que precisen complementar su ahorro doméstico y financiar el déficit de su balanza corriente. Pero también nos muestra hasta qué punto tales movimientos son sensibles a las variaciones de expectativas, tanto derivadas de los «desequilibrios fundamentales» como de la percepción de debilidad o inconsistencia en las medidas de política económica aplicadas; y en qué grado puede resultar inestable un sistema que pretenda conciliar tipos de cambios fijos y liberalización total de los movimientos de capital, a menos que los países estén dispuestos a renunciar a la independencia de su política monetaria.

Dejando de lado soluciones que pretendan introducir restricciones en los mercados de capital (en contra de la actual preferencia por el funcionamiento libre de los mercados y cuya eficiencia resulta muy dudosa), la experiencia reciente hace difícil de imaginar la introducción de cualquier sistema que mejore considerablemente la estabilidad y el nivel de exigencias del actual sistema monetario internacional. De las alternativas descritas, el sistema de las zonas-objetivo es el que parece ofrecer un margen de flexibilidad considerable que probablemente permitiria hacerlo compatible con la movilidad actual de capitales y la globalización de los mercados financieros. Pero su introducción exigiría acuerdos de coordinación de las políticas monetarias que implican renunciar parcialmente a la independencia financiera. Las últimas experiencias en el G-7, no hacen concebir 
esperanzas a este respecto. Tampoco resultan esperanzadores los intentos de coordinación en la Unión Europea, pese al menor número y mayor convergencia de sus economías.

Todo lo señalado apunta a la continuidad del sistema monetario internacional actual (o si se quiere, del «no-sistema» que padecemos). No hay duda que dicho sistema dista mucho de ser ideal, pero no se ve en el horizonte nada que pueda sustituirlo y, al mismo tiempo, ser capaz de acomodar la expansión e integración creciente de los mercados financieros. Ello sólo sería posible con una mejora dramática en la coordinación económica, difícilmente previsible en un futuro próximo.

\section{BIBLIOGRAFÍA}

ALONSO, J., y LINDE, L. M. (1993): «Mercados de divisas y crisis cambiarias: una nota a propósito del informe del Grupo de los Diez», Fundación FIES, Cuadernos de Información Económica, n." 76-77.

Bercisten, C. F., y WILLIAMSON, J. (1983): «Exchange rates and trade policy», en W. R. Cline (ed.), Trade Policy in the 1980's, Washington: Institute for International Economics.

CROCKETT, A., y GOLDSTEIN, M. (1987): «Strengthening the International Monetary System: exchange rates, surveillance, and objective indicators», Occasional Paper, IMF, n." 50.

DAvidson, P. (1992-93): «Reforming the world's money», Journal of Post-Keynesian Economics, vol. 15, n." 2 .

Domingulz, K. M., y Frankel, J. A. (1993): Does Foreign Exchange Intervention Work?, Institute for International Economics, Washington, D.C.

EICHENGREEN, B, y WYPLOSZ, C. (1993): «The unstable EMS», Brooking Papers on Economic Activity, 1 ,

Eichengrien, B.; TOBin, J., y Wyplosz, C. (1995): «Two cases for sand in the wheels of international finance», en The Economic Journal, vol. 105, n." 428.

Frenkel, J. A., y Goldstein, M. (1986): «A guide to target zones», Staff Papers, IMF, vol. $33, n . " 4$.

Friedman, M. (1953): Essays in Positive Economics, University of Chicago Press, Chicago.

GARBER, P., y SVENSSON, L. E. O. (1994): «The operation and collapse of fixed exchange rate regimes», en NBER Working Paper Series, n." 4.971.

GARBER, P., y TAYLOR, P. M. (1995): «Sand in the wheels of foreign exchange markets: a sceptical note», en The Economic Journal, vol. 105, n." 428.

JURGENSEN, P. (1983): Report of the Working Group on Exchange Market Intervention. U.S. Treasury Department, Washington.

INTERNATIONAL MONETARY Fund, ReSEARCH DEPARTMENT (1984): «The exchange rate system: Lessons of the past and options for the future», Occasional Paper, IMF, n." 30.

Kenen, P. B. (1995): «Capital controls, the EMS and EMU», en The Economic Journal, vol. 105, n. $^{\circ} 428$.

KRUGMAN, P. (1991): «Target zones and exchange rate dynamics», en Quarterly Journal of Economics, n." 106. 
LINDE, L. M. (1993): «Las medidas del Banco de España de septiembre y octubre de 1992 penalizando la especulación cambiaria», en Papeles de Economía Española, n." 54.

MEADE, J. E. (1955): «The case for variable exchange rates», en Three Banks Review, vol. 27. MELTZER, A. H. (1993): «Real exchange rates: Some evidence from the postwar years», en Review, Federal Reserve Bank of St. Louts, marzo/abril, vol. 75, n." 2.

MCKINNON, R. I. (1988): «Monetary and exchange rates policies for international financial stability: a proposal», Journal of Economic Perspectives, 2.

Mussa, M.; Goldostein, M.; Clark, P. B.; Mathieson, D. J., y Bayoumi, T. (1994): «Improving the International Monetary System, Constraints and Possibilities», Occasional Paper, IMF, n." 116.

OBSTFELD, M. (1986): «Rational and self fulfilling balance of payments crises», en American Economic Review, vol. 76.

PADOA-SchiopPA, T. (1988): «The European Monetary System: a long-term view», en Giavazzi, F.; Micossi, S., y Miller, M. (eds.): The European Monetary System, Cambridge University Press, Cambridge.

PORTER, R. (1993): «EMS and EMU after the fail». The World Economy, vol. 16, n." 1.

RoOT, F. R. (1994): International trade and investment, South-Western Publishing Co., Cincinnati, Ohio. Seventh Edition.

SANTILlán, J. (1994): «El SME, los mercados de divisas y la transición hacia la Unión Monetaria», Banco de España, Servicio de Estudios, Documento de trabajo n." 9.421.

SMAGHi, L. B., y Micossi, S. (1990): «Monetary and exchange rate policy in the EMS with free capital mobility», en DE GRAUWE, P., y PAPADEMOS, L. (eds.): The European Monetary System in the 1990's, Longman Group.

TOBIN, J. (1978): «A proposal for international monetary reform», Eastern Economic Journal, vol. 4. Reproducido en J. TobIN, Essays in Economics (Cambridge Macs, MIT Press, 1982).

VARELA, F. (1994a): «La era de Bretton Woods: la experiencia de los tipos de cambio fijos», en El Fondo Monetario Internacional, el Banco Mundial y la economía española, coordinado por Manuel Varela, ed. Pirámide.

- (1994b): «Movilidad de capitales y tipos de cambio ajustables: de Bretton Woods al Sistema Monetario Internacional», en Economistas, n. "61.

VARLla PARACHE, F. y M. (1974): El Sistema Monetario Internacional. Presente y Futuro, Ed. Planeta, Barcelona.

VIÑALS, J. (1986): «Hacia una menor flexibilidad de los tipos de cambio en el sistema monetario internacional?», en Papeles de Economia Española, n." 28.

WILliamson, J. (1983): The Exchange Rate System, Institute for International Economics, Washington D.C.

- (1990): «The "blueprint" proposals for international monetary reform», en Changing Exchange Rate Systems, dirigido por Christopher Johnson, Pinter Publishers, Londres.

WII.LIAMsON, J., y MILLER, M. H. (1987): Targets and Indicators: A Blueprint for International Coordination of Economic Policy, Institute for International Economics, Washington D.C.

Williamson, J., y Milner, C. (1991): The World Economy: A Text Book in International Economics, Harvester-Wheatsheaf, Cambridge, Gran Bretaña.

WyPLosz, C. (1988): «Capital flow liberalization and the EMS: A French perspective». European Economy, n." 36. 